\title{
CMBE Moves to the Structured Abstract Format: A Note from the Editor
}

After discussion among the Board of Associate Editors, the Cellular and Molecular Bioengineering journal has decided to transition to a structured abstract style. The term "structured abstract" refers to the style in which separate sections such as Introduction, Methods, Results, and Conclusions are broken out in the abstract. The style was originally developed in the 1980s and 1990s to help health professionals gain a concise summary of a published study, and to aid in computerized searches of the scientific literature. Roughly $30 \%$ of new abstracts added to MEDLINE in PubMed are structured, and over three million structured abstracts are currently indexed in PubMed (https://www.nlm.nih.gov/bsd/policy/structured_abstr acts.html).

We do not enter into a change of this nature without some reservations. For example, there is some concern among our associate editors that the structured abstract style is not a natural fit for computational and theoretical studies, and seems counter to the trend of some journals to relegate the Methods section of papers to the end of main text. One suggestion was to make the Methods section of the abstract optional for authors. Other associate editors for our journal note that the structured abstract is already common in many pharmaceutical and biomaterials journals, and other journals that they read and publish in. As one associate editor noted, the structured abstract format becomes more appealing over time, "since every primary research paper describes a body of work that was done for a reason, involved doing something, revealed something and led to inferences, regardless of the order/layout of the manuscript."

While several different structured abstract formats exist, for instance some including sections titled "Objectives" and "Discussion", in Cellular and Molecular Bioengineering we will require authors include the following four separate sections in their abstracts: Introduction, Methods, Results, and Conclusions. We hope that this change will help to bring our work to a broader biomedical audience, and you will soon start to see CMBE papers featuring this new structured abstract. As an editorial board, we will continue to monitor and reexamine the effectiveness of our publication policies, so that they may best serve the needs of bioengineering researchers and the Biomedical Engineering Society.

Thank you for publishing in and reading our journal, and don't forget to follow our latest developments on Facebook (www.facebook.com/CMBEjournal) and Twitter (www.twitter.com/CMBEjournal)!

Michael R. King

Department Chair of Biomedical Engineering

Vanderbilt University

Electronic mail: mike.king@vanderbilt.edu 